See discussions, stats, and author profiles for this publication at: https://www.researchgate.net/publication/286108694

\title{
Fast generation of LULC maps for temporal studies in North-Western Africa
}

Conference Paper · July 2014

DOI: 10.1109/IGARSS.2014.6947435

CITATION

1

5 authors, including:

(a) Pol del Aguila Pla

École Polytechnique Fédérale de Lausanne

14 PUBlications 17 CITATIONS

SEE PROFILE

(a)

J. Marcello

Universidad de Las Palmas de Gran Canaria

76 PUBLICATIONS 691 CITATIONS

SEE PROFILE

\section{READS}

32

Felipe Calderero

University Pompeu Fabra

20 PUBLICATIONS 250 CITATIONS

SEE PROFILE

Francisco Eugenio

Universidad de Las Palmas de Gran Canaria

69 PUBLICATIONS 599 CITATIONS

SEE PROFILE

Some of the authors of this publication are also working on these related projects:

Inference on Quantised Data View project

Project Automated Rodent EEG Sleep Analysis with Machine Learning Methods View project 


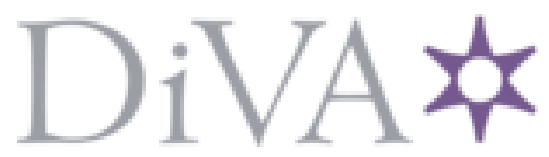

http://www.diva-portal.org

\section{Postprint}

This is the accepted version of a paper presented at Joint International Geoscience and Remote Sensing Symposium (IGARSS 2014) / 35th Canadian Symposium on Remote Sensing (35th CSRS)..

Citation for the original published paper:

del Aguila, P. (2014)

Fast generation of LULC maps for temporal studies in North-Western Africa.

In: IEEE International (ed.), IGARSS 2014: Geoscience and Remote Sensing Symposium (pp. 4280-4283).

http://dx.doi.org/10.1109/IGARSS.2014.6947435

N.B. When citing this work, cite the original published paper.

Permanent link to this version:

http://urn.kb.se/resolve?urn=urn:nbn:se:kth:diva-157117 


\title{
FAST GENERATION OF LULC MAPS FOR TEMPORAL STUDIES IN NORTH-WESTERN AFRICA
}

\author{
del Aguila, $P^{1}{ }^{1}$, Calderero, $F^{2}{ }^{2}$ Marqués , $F^{3}{ }^{3}$ Marcello, ${ }^{4}{ }^{4}$; Eugenio, $F^{4}{ }^{4}$ \\ 1: Signal Processing Lab, KTH. 2: Image Processing Group, UPF. 3: Image Processing Group, UPC. \\ 4: Institute for Oceanography and Global Change, ULPGC.
}

\begin{abstract}
This paper provides an objective evaluation of six supervised classification techniques and three state of the art features, with the objective of obtaining a single combination of them that provides both robustness and objective performance improvements. As a conclusion, a simple procedure for obtaining LULC maps with four targeted classes is proposed.
\end{abstract}

Index Terms - LULC, robust, empirical evaluation

\section{INTRODUCTION}

LULC (Land-Use/Land-Cover) maps provide information that is potentially useful for many applications. Tracking of natural catastrophes, such as fire or tornadoes, could be significantly aided by these maps, were they obtained at the proper rate. Even at the present day, when LULC maps are often automatically obtained from remote sensing multispectral images, the rate at which this is done greatly limits the aforementioned applications. Controversially, this limit is not caused by technology or technique, but by the approach researchers have taken towards LULC maps' generation. In order to leverage for the high costs of paid-for remote sensing imagery, the academic community in this field has tended towards the design of image-, satellite-, region- or sensor-specific algorithms that attempt to achieve maximal classification performance. In other words, neither generality nor the celerity with which the LULC map is obtained have been design parameters in LULC classification studies. As a consequence, most studies use either a reduced number of images or a reduced number of different satellites. This generally results in conclusions that are only applicable to very specific situations $[1,2,3,4,5]$. While this is not necessarily a problem, it tends to bias the synthesis process, often resulting in studies with opposite conclusions [2,5]. In an attempt to make a small step against this methodological flaw, this publication presents results based on 6 images from 3 different satellites and 2 different regions.

We would like to thank the TELECAN project (MAC/3/C181: PCTMAC 2007/2013) for the provision of the SPOT imagery, as well as the USGS for the provision of LANDSAT products.
The robustness of a (statistical) algorithm is already of intrinsic value. In the particular application of LULC classification, however, it is of special relevance. A robust LULC classification algorithm, even if it is a supervised one, could allow for the use of LULC maps in the event of natural catastrophes and in other time dependent situations.

This article will present the results of an empiric study aimed at obtaining a robust LULC classification algorithm. In Section 2, the methodology that has been followed is presented. Particularly, Subsection 2.1 describes precisely the considered dataset, Subsection 2.2 motivates the choice of the classification methods under analysis and their parameters, and Subsection 2.3 states the procedure followed during experimentation. Section 3 exposes the experimental results, and details the obtained conclusions.

\section{METHODOLOGY}

\subsection{Dataset description}

The dataset under study is formed by the images shown in Figure 1 and Figure 2. Note that these images are diverse in several aspects. The first group comprises 4 (four) images of Gran Canaria island, obtained by a total of 3 (three) different satellites. This group constitutes a long temporal series, since it contains images obtained from 1984 to 2009 . The second group comprises 2 (two) images of the same region of Senegal, both captured by the LANDSAT 5 satellite. However, this group also constitutes a long temporal series, being the first image captured in 1985 and the second in 2010. The fact that these two regions have extremely different properties is clear. These differences make one of the regions much easier to classify than the other, fact that will be utilized during the experimental procedure design (see Subsection 2.3).

Note also that the satellites that have obtained these images have a variety of different features, ranging from $10 \mathrm{~m}$ to $30 \mathrm{~m}$ in spatial resolution, and from three to seven bands in spectral resolution. The detailed specifications of each satellite are included in Table 1.

Aside from all the previous diversity, note that within the presented study data there is a substantial variety of surfaces, ranging from wild vegetation to highly urbanized territories. 
In this aspect, it is the authors' belief that an algorithm that consistently performs across the presented dataset can be considered robust, and is therefore a suitable candidate to obtain LULC maps from any remote sensing image without further modification.

Without loss in the generality of the study, the whole dataset was preprocessed, as it is usual in the field, by applying the appropriate radiometric calibration and performing an atmospheric correction based on the $6 \mathrm{~S}$ model $[6,7]$.

\begin{tabular}{c|c} 
Satellite & Bands and spatial resolution \\
\hline \hline SPOT 1 & $\mathrm{R}, \mathrm{G}, \mathrm{NIR} @ 20 m$ \\
\hline SPOT 5 & $\mathrm{R}, \mathrm{G}, \mathrm{NIR} @ 10 \mathrm{~m}, \mathrm{SWIR} @ 20 \mathrm{~m}$ \\
\hline LANDSAT 5 & $\mathrm{R}, \mathrm{G}, \mathrm{B},(\mathrm{N}, \mathrm{S}, \mathrm{HS}, \mathrm{LW}) \mathrm{IR} @ 30 \mathrm{~m}$
\end{tabular}

Table 1. Spectral bands and spatial resolution for different sensors. B, G, R: Blue, Green, Red. NIR, SWIR, LWIR: Near, Short Wave, Long Wave Infrared. HSWIR: Upper part of the SWIR band.

\subsection{Classifier and feature pre-selection}

There are many different approaches to classify remote sensing imagery [8]. Therefore, a pre-selection had to be done before the experimental phase. The 6 classifiers that were finally tested were selected based on their historical relevance in the field. On the other hand, their availability as already implemented modules within ENVI version 5.0 (Exelis Visual Information Solutions, Boulder, Colorado) was also considered as a factor, since this is the software platform that has been used in this study. It is worthwhile to note that the implementations of some of the methods within this software platform are not entirely standard, and therefore, some of the results presented here will slightly vary from what can be obtained by other specific implementations.

With these criteria, the classifiers selected for testing were the algorithms known as: Support Vector Machines (SVM), Neural Networks (NN), Minimum Mahalanobis Distance, Maximum Likelihood, Minimum Distance and Parallelepipeds. For details on any of them or their applications to remote sensing, please refer to [8].

While most of these classifiers are non parametric, both the NN and the SVM algorithms are known to depend heavily on their parameters. In both cases, many studies concerning parameter selection can be found in the literature. In the particular case of the NN algorithms, however, these studies always result on complex heuristic processes that are not easily automated [9]. Consequently, the NN parameter optimization lays beyond our goals, and the values chosen by the heuristics built into ENVI were used. In order to avoid overfitting and limit the experimentation time, the only constraint established upon the NN classifiers was that the neural network could not have more than one hidden (intermediate) layer.

On the other hand, the SVM kernel and parameter selection was done according to the conclusions in [10], which states that, for LULC classification, the RBF (Radial Basis Functions) kernel is a suitable choice, and that its parameters should be selected by an exponential grid search. In Section 3 , the results of applying this procedure on one of the images is shown.

The selection of the three different features was done according to their popularity in the field and their applicability to our specific problem. The NDVI (Normalized Difference Vegetation Index) and the MNDWI (Modification of the Normalized Difference Water index) were the two first features. Their tight relation to the physical properties of the spectrum and the fact that they were specifically designed to distinguish two of the four considered classes, i.e. Vegetation, Buid-Up Land, Bare Soil and Water, made them obvious choices. In order to account for the high number of studies dealing with textural or multi-scale features, e.g. $[1,5]$, the mean band entropy within a square region, a textural feature, was also introduced. The size of the region (in square meters) was selected through visual assessment of the compromise between the resulting variance in the sea areas and the loss of information in land areas. It was finally set to $330 \mathrm{~m} \times 330 \mathrm{~m}$, producing results as those shown in Figure 1.

\subsection{Experimental procedure}

As it was mentioned in Subsection 2.1, the two different considered regions are not equally hard to classify. Gran Canaria, in the Canary Islands, Spain, is characterized by its steep orography and intertwined land classes, while the considered Senegalese region tends to have clean and sharp edges between the different classes (see details of specific regions in Figure 2). The latter is therefore much easier to classify. Since the performed experimentation needed to be extensive, and classification of remote sensing imagery is time consuming and costly, only two of the images were used for intensive experimentation. The remaining images were reserved for verification purposes only.

Controversially, both of the images used for intensive experimentation were from the region of Gran Canaria. This was done under the hypothesis that since Gran Canaria was a highly variant and diverse region, i.e. a hard region to classify, statistically relevant improvements in performance on its classification would imply generic algorithm improvements. Specifically, the two selected images were image A (SPOT 5, 2009/08/01) and B (LANDSAT 5, 1984/09/22).

The set of considered classes was selected to roughly match a level I Anderson classification [11] on the targeted region. For each region, two different databases were generated with labeled points for each class. Note that this was done in such a manner that these points did not change their class assignation throughout the considered period of time. The first of these databases, which we will refer as train database, includes a higher number of points, drawn from polygons defined over the regions, and therefore includes 


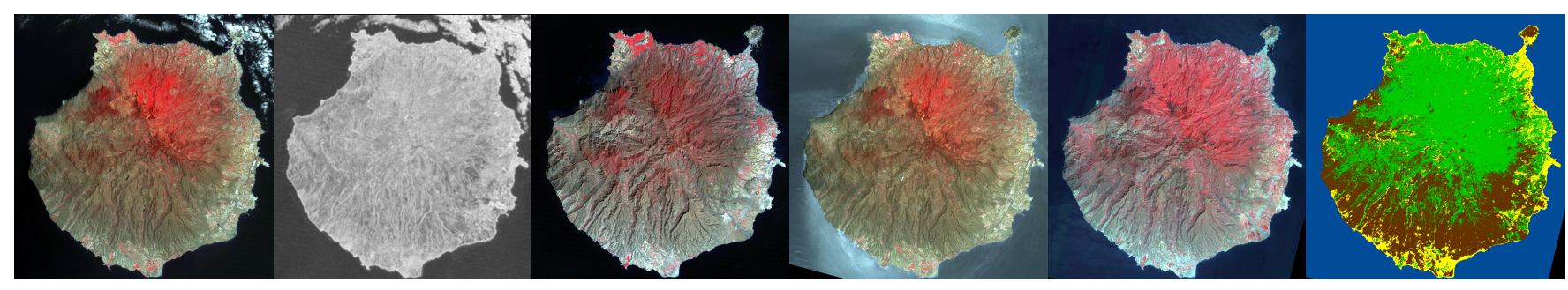

Fig. 1. From left to right: LANDSAT 5, $9^{\text {th }}$ of July 2009 and its mean spatial entropy computed with a $330 \mathrm{~m} \times 330 \mathrm{~m}$ window; LANDSAT 5, 22 ${ }^{\text {nd }}$ of September 1984 (B); SPOT 5, $1^{\text {st }}$ of August 2009 (A); SPOT 1, $25^{\text {th }}$ of March 1988 and its LULC map, computed by the finally selected combination features - classifier.

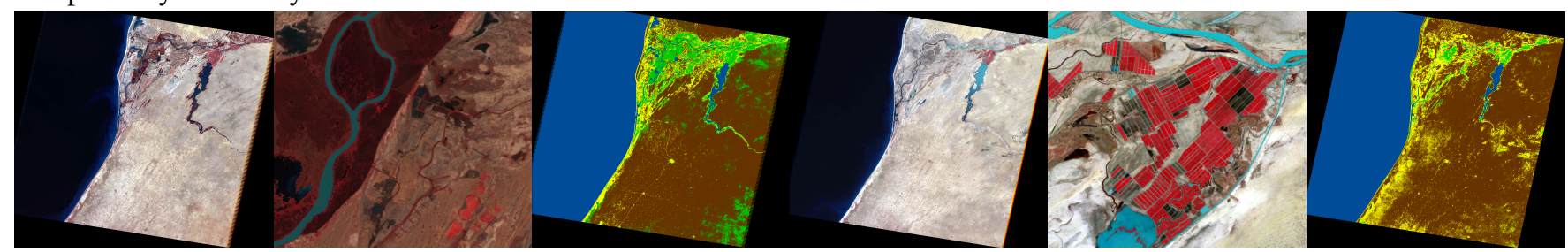

Fig. 2. From left to right: LANDSAT 5, $26^{\text {th }}$ of January 2010, a detail of one of its regions, and its final classification; LANDSAT 5, $21^{\text {st }}$ of January 1985, a detail of one of its regions, and its final classification.

some unclear or class-border points. The second database, to be referred as test database, includes only 100 (a hundred) points per class, chosen in a purely random manner and therefore distributed across the whole image. Note that, if we define the classification accuracy as the proportion of properly classified points in a database, this measure will not bias our conclusions towards classifiers that describe more accurately the most frequent classes, since the test database contains the same number of points in each class.

The experimentation was divided in several phases. In the first phase, all classifiers were tested on images A and B. Using these results, only the two consistently better classifiers were kept for the second phase, in which the selected features were tested with both of them. In the third phase, the three best performing combinations were tested in the remaining images from Gran Canaria. Finally, only the best combination in the third phase was tested on the images from Senegal, in order to verify its robustness, and therefore its suitability to the task.

\section{RESULTS AND CONCLUSIONS}

As mentioned in Subsection 2.2, the SVM RBF classifier parameters were tunned by an exponential grid search, as described in [10]. The results of applying this procedure to image A are shown in Figure 3. This figure illustrates the unexpected conclusion that the width parameter of the RBF kernel is irrelevant, within a range from $\gamma=2^{-5}$ to $\gamma=2^{3}$. On the other hand, the optimal regularization parameter was found to be $C=1024$.

The results of the procedure described in Subsection 2.3 are shown in Table 2. There we can observe that, coherently with what has been reported across the state of the art
[12, 10, 13], SVM algorithms tend to yield better results in LULC classification tasks. On the other hand, our results also suggest that the addition of the classical nonlinear indices (NDVI, MNDWI) does not improve performance when using advanced, nonlinear machine learning techniques such as SVM or NN. Finally, we can observe that the obtained combination is robust, since it yields consistently high accuracies over the considered images.

Conclusively, we propose the SVM RBF classifier (any $\gamma, C=1024)$, operating with the spectrum and the mean band entropy as features, as a standard LULC classification technique for cases in which parameter optimization is not an option.

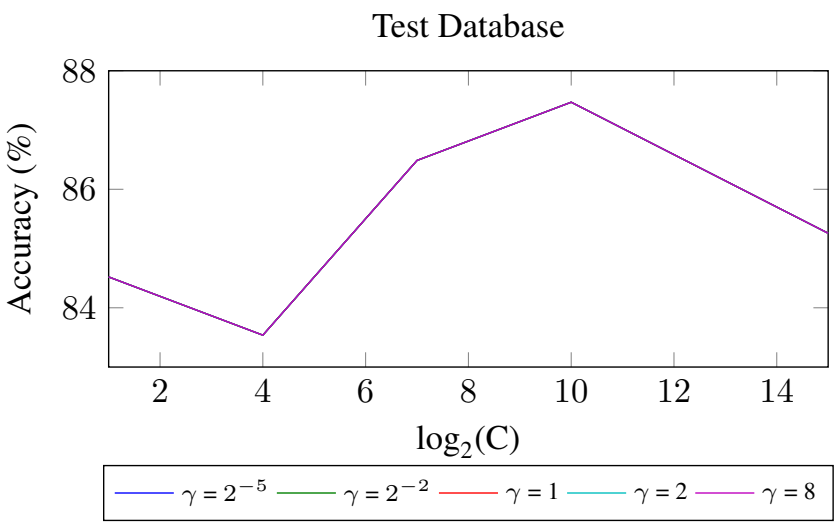

Fig. 3. Search on an exponential grid $\left(2^{n}\right)$ of the optimal parameters for the SVM algorithm with RBF kernel ( $C$ and $\gamma$ ) on image A before any preprocessing. The optimal was found for $C=1024$ and any $\gamma$. 
Gran Canaria

\begin{tabular}{|c|c|c|c|c|}
\hline Classifier and Features / Image & \multicolumn{2}{|c|}{ SPOT 5, $2009(\mathrm{~A})$} & \multicolumn{2}{|c|}{ LANDSAT 5, $1984(\mathrm{~B})$} \\
\hline Parallelepipeds & $46.50 \%$ & $45.60 \%$ & $40.83 \%$ & $45.13 \%$ \\
\hline Minimum Distance & $68.00 \%$ & $67.02 \%$ & $63.00 \%$ & $69.97 \%$ \\
\hline Min. Mahalanobis Distance & $84.50 \%$ & $82.35 \%$ & $84.17 \%$ & $85.68 \%$ \\
\hline Max. Likelihood & $86.50 \%$ & $91.22 \%$ & $80.17 \%$ & $90.05 \%$ \\
\hline $\mathrm{NN}$ & $91.33 \%$ & $94.90 \%$ & $85.83 \%$ & $94.98 \%$ \\
\hline SVM & $93.67 \%$ & $96.21 \%$ & $88.67 \%$ & $94.06 \%$ \\
\hline $\mathrm{NN}+\mathrm{NDVI}$ & $87.33 \%$ & $93.89 \%$ & $25.00 \%$ & $25.58 \%$ \\
\hline SVM + NDVI & $93.5 \%$ & $96.13 \%$ & $89.33 \%$ & $93.93 \%$ \\
\hline NN + MNDWI & $87.66 \%$ & $87.02 \%$ & $25.00 \%$ & $25.58 \%$ \\
\hline SVM + MNDWI & $92.83 \%$ & $96.27 \%$ & $89.33 \%$ & $93.93 \%$ \\
\hline NN + Mean Entropy & $84.67 \%$ & $91.28 \%$ & $87.5 \%$ & $91.27 \%$ \\
\hline \multirow[t]{2}{*}{ SVM + Mean Entropy } & $92.17 \%$ & $97.85 \%$ & $92.33 \%$ & $96.25 \%$ \\
\hline & Test & Train & Test & Train \\
\hline \multicolumn{5}{|l|}{ Gran Canaria } \\
\hline Classifier / Image & \multicolumn{2}{|c|}{ SPOT 1,1988} & \multicolumn{2}{|c|}{ LANDSAT 5, 2009} \\
\hline SVM & $82.83 \%$ & $84.13 \%$ & $86.33 \%$ & $91.23 \%$ \\
\hline SVM + MNDWI & $86.00 \%$ & $89.77 \%$ & $84.83 \%$ & $91.10 \%$ \\
\hline \multirow[t]{2}{*}{ SVM + Mean Entropy } & $90.67 \%$ & $92.29 \%$ & $87.67 \%$ & $93.37 \%$ \\
\hline & Test & Train & Test & Train \\
\hline \multicolumn{5}{|l|}{ Senegal } \\
\hline Classifier / Image & \multicolumn{2}{|c|}{ LANDSAT 5, 1985} & \multicolumn{2}{|c|}{ LANDSAT 5, 2010} \\
\hline SVM + Mean Entropy & \multicolumn{2}{|c|}{$97.86 \%$} & \multicolumn{2}{|c|}{$96.38 \%$} \\
\hline & \multicolumn{2}{|c|}{ Test } & \multicolumn{2}{|c|}{ Test } \\
\hline
\end{tabular}

Table 2. Accuracy over the different images for each combination classifier - feature. The performance over the training database is provided merely to evaluate overfitting. In all cases, the specified classifiers were trained and evaluated on the images' spectrum and the feature after the + symbol, when applicable.

\section{REFERENCES}

[1] Yanchen Bo, "Improve the accuracy of classified land use map by exploiting the multi-scale properties of the remotely sensed data," in Proceedings of SPIE, the International Society for Optical Engineering. Society of Photo-Optical Instrumentation Engineers, 2007, pp. 679034-1.

[2] Hanqiu Xu, "Extraction of urban built-up land features from landsat imagery using a thematic-oriented index combination technique," Photogrammetric Engineering and Remote Sensing, vol. 73, no. 12, pp. $1381,2007$.

[3] Fauvel Mathieu, Chanussot Jocelyn, Benediktsson Jón Atli, et al., "Kernel principal component analysis for the classification of hyperspectral remote sensing data over urban areas," EURASIP Journal on Advances in Signal Processing, vol. 2009, pp. 1-14, 2009.

[4] Offer Rozenstein and Arnon Karnieli, "Comparison of methods for land-use classification incorporating remote sensing and gis inputs," Applied Geography, vol. 31, no. 2, pp. 533-544, 2011.

[5] Guiying Li, Dengsheng Lu, Emilio Moran, and Scott Hetrick, "Landcover classification in a moist tropical region of brazil with landsat thematic mapper imagery," International journal of remote sensing, vol. 32, no. 23, pp. 8207-8230, 2011.

[6] Svetlana Y Kotchenova, Eric F Vermote, Raffaella Matarrese, Frank J Klemm Jr, et al., "Validation of a vector version of the $6 \mathrm{~s}$ radiative transfer code for atmospheric correction of satellite data. part i: Path radiance," Applied Optics, vol. 45, no. 26, pp. 6762-6774, 2006.
[7] Svetlana Y Kotchenova and Eric F Vermote, "Validation of a vector version of the $6 \mathrm{~s}$ radiative transfer code for atmospheric correction of satellite data. part ii. homogeneous lambertian and anisotropic surfaces," Applied Optics, vol. 46, no. 20, pp. 4455-4464, 2007.

[8] Paul Mather and Brandt Tso, Classification methods for remotely sensed data, CRC press, 2003.

[9] Christopher M Bishop, Neural networks for pattern recognition, Oxford university press, 1995.

[10] Taskin Kavzoglu and I Colkesen, "A kernel functions analysis for support vector machines for land cover classification," International Journal of Applied Earth Observation and Geoinformation, vol. 11, no. 5, pp. 352-359, 2009.

[11] Robert A Schowengerdt, Remote sensing: models and methods for image processing, Academic press, 2006.

[12] Paolo Mantero, Gabriele Moser, and Sebastiano B Serpico, "Partially supervised classification of remote sensing images through svm-based probability density estimation," Geoscience and Remote Sensing, IEEE Transactions on, vol. 43, no. 3, pp. 559-570, 2005.

[13] Giorgos Mountrakis, Jungho Im, and Caesar Ogole, "Support vector machines in remote sensing: A review," ISPRS Journal of Photogrammetry and Remote Sensing, vol. 66, no. 3, pp. 247-259, 2011. 